\title{
Trends in Age Distributions, Complications, Hospital Stay, and Cost Incurred in a Chinese Diabetic Population from 2006 to 2013
}

Zuolei Dai, Xin Zhang, Hongwei Shan and Yun Liu*

The First Affiliated Hospital of Nanjing Medical University, Nanjing 210029, China

\begin{abstract}
Diabetes is becoming a major chronic disease which affects human health. The diabetic data in hospital of large amount contains much information. Here we analyzed the in hospital diabetes's data and hope to provide basic data and scientific basis for the control of diabetes and the decrease of economic burden of diabetics in China Mathematic statistical methods were made to the in hospital diabetics' data. Retrospective analysis was made to the age, gender, days in hospital, cost and complications. The result shows that more and more young people have diabetes, and the number of diabetics is growing. The average days in hospital of diabetics is increasing along with age. Average number of complications is 3.108 . Hospital costs are getting higher along with the number of complications. The average days in hospital decreases from 14.15 in 2006 to 10.61 in 2013 . Hospital costs presented an increasing trend, so does daily hospital costs. This study resulted in a fact that age, gender, days in hospital, cost and complication of diabetics has impacts on each other. Cost is directly influenced by the number of complications and the days in hospital. People in China should pay attention to the prevention of diabetes, and diabetics should follow the doctor's advice to control blood sugar to normal state.
\end{abstract}

Keywords: Diabetes; Inpatient data; Analysis; Prevention

\section{Introduction}

The prevalence of chronic diseases has been rising since 1980s as people's living standard gets higher and the pace of modern life gets faster. Chronic diseases have become a major threat to people's health, accounting for $80 \%$ of the death toll [1]. Diabetes is the most common metabolic diseases. Its incidence has been increasing over the past decades [2]. In 2013, the rate of diabetes has been up to $9.6 \%$ in China and $9 \%$ all over the world. Studies show that the prevalence rate of diabetes in China will reach $13 \%$ by the year 2035 [3-5]. Diabetes has become an important factor that endangers human health. Medical cost and labor loss caused by diabetes have laid enormous economic burden on the patients [6-8]. Cost on diabetes accounts for about $13 \%$ of the cost on health service in China while $5 \%-6 \%$ in developed countries. The direct cost for type 2 diabetes in China in 2007 is about 26 billion US dollars and will reach 47.2 billion US dollars in 2030 [9]. Therefore, under the condition of the increasing rate of diabetes, the mechanism of follow-up survey on diabetics should be set up in China, in which includes the statistics on the amount of cost on health and fully implementation of perfect survey on health economics. This research collected the clinical data of in hospital diabetics in the First Affiliated Hospital of Nanjing Medical University and analyzed the basic situation of diabetes. The exploration of the changes and the analysis of the reasons provide scientific basis for the clinical treatment and hospital management. They also offer the references for the prevention of local diseases and health service in China.

\section{Methods}

The data we used is directly extracted from in hospital records. All the patients this research studied is hospitalized patients. Using principal diagnoses of hospitalized patients, diseases cases are selected to establish a database. By counting the times of patients be in hospital for diabetics, we gained the percentage of times of diabetics out of the whole inpatients. Gender distribution is gained by counting gender of diabetics. Using the same method, age distribution in different groups comes out. In hospital days and hospital costs are calculated, so as to get daily costs on average. Through the combination with the age distribution, we got the distribution of in hospital days in different age groups. According to the data of other diagnosis, we got the situation of diabetic complications. We also analyzed the mean number of complications of diabetics.

\section{Results}

\section{General review}

During the years from 2006 to 2013, there were 531,718 in hospital patients, in which males accounted for $49.36 \%$ and females for $50.64 \%$. The number of diabetics was 12,214 , accounting for $2.30 \%$ of the total in hospital patients. Of all the 12,214 diabetics, $57.30 \%$ were males and $42.70 \%$ were females.

\section{Age analysis}

We divided the diabetics into five groups by every 20 years old. Age distribution of diabetics in different age-groups shows as Figure 1. All the five groups show an increasing trend. Diabetics between 41 and 80 years old occupied over $80 \%$ of the whole diabetics in all the eight years. Chi-square test is used on the number of 1-20 years old diabetics and over 20 years old diabetics. The result shows there is significant difference $(\mathrm{p}<0.001)$ between the numbers of the two groups of diabetics. The number of 1-20 years old group has faster average growth in the eight years, which is $25.34 \%$, than the over 20 years old group, which is $14.52 \%$.

*Corresponding author: Yun Liu, Department of Information, The First Affiliated Hospital of Nanjing Medical University, Nanjing 210029, China, Tel: 86-2583781379; E-mail: liuyun@njmu.edu.cn

Received February 11, 2015; Accepted April 06, 2015; Published April 10, 2015

Citation: Dai Z, Zhang X, Shan H, Liu Y (2015) Trends in Age Distributions, Complications, Hospital Stay, and Cost Incurred in a Chinese Diabetic Population from 2006 to 2013. J Bioanal Biomed 7: 050-053. doi:10.4172/1948-593X.1000123

Copyright: (c) 2015 Dai Z, et al. This is an open-access article distributed under the terms of the Creative Commons Attribution License, which permits unrestricted use, distribution, and reproduction in any medium, provided the original author and source are credited. 
Citation: Dai Z, Zhang X, Shan H, Liu Y (2015) Trends in Age Distributions, Complications, Hospital Stay, and Cost Incurred in a Chinese Diabetic Population from 2006 to 2013. J Bioanal Biomed 7: 050-053. doi:10.4172/1948-593X.1000123

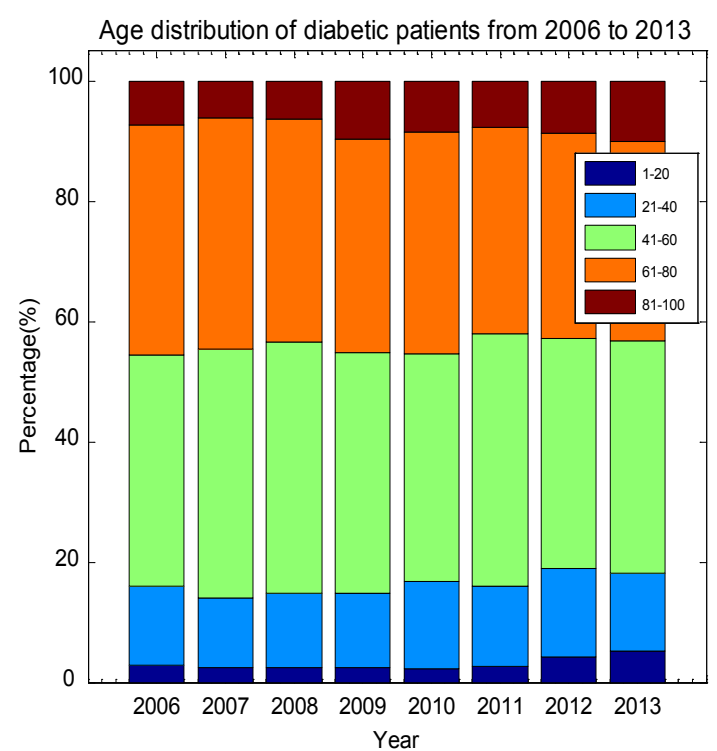

Figure 1: Age distribution of diabetics from 2006 to 2013.

Horizontal axis represents the years from 2006 to 2013. Vertical axis represents the proportion of diabetics in each year. Different colors represent different age-groups.

\section{Hospital stay analysis}

Hospital stay (days patient stay in hospital) of diabetics in different age-groups shows as Figure 2a. The in hospital days of diabetics in different age groups are counted. The number of diabetics in hospital for 8 days is the largest. Most patients (81.83\%) tend to stay in hospital for 4 to 16 days. Diabetics between 41-60 years old have the longest hospital stay.

Average hospital stay of diabetics in different age groups is generated by dividing the total days by the number of total diabetics of different age groups and is shown as Figure 2b. Mean hospital stay increases with age, especially for the diabetics of 81-100 years old. Meanwhile, we noted that in hospital days of diabetics of 1-10 years old (9.8 days) are more than one day longer than those of 11-20 years old (8.1 days).

\section{Cost analysis}

We analyzed the total cost on diabetes and total diabetics number of each year form 2006 to 2013. In hospital costs of diabetics in different age group shows as Figure 3a. Diabetics between $81-100$ years old have the most in hospital cost, nearly half of the whole diabetics' cost of each year. In hospital costs of diabetics under 80 years old shows an increasing trend, although there is a fluctuation in both 2011 and 2013.

By analyzing in hospital days and cost we get the average hospital cost of diabetics per day which is shown in Figure $3 \mathrm{~b}$. The cost of each diabetic is 508 RMB per day in 2006, and increases year by year to 930 $\mathrm{RMB}$ per day in 2013. The average growth of cost in the eight years is 9.02\%.

\section{Complications}

To see how complications influence costs incurred, we analyzed the complications of diabetics. The number of other diagnostic data shows as Table 1 . In all 12,214 cases of hospitalized patients, there are 10,086 patients who have complications, accounting for $82.58 \%$, most of which ( 3,039 cases) have one complication, accounting for $24.88 \%$. The number of complications of all patients is 37,968 , averaging 3.108 complications per patient. Table 2 lists the most 9 complications.

Daily hospital costs show as Figure 4. Diabetes without complications spends 5,866.6 RMB on average. However, diabetics

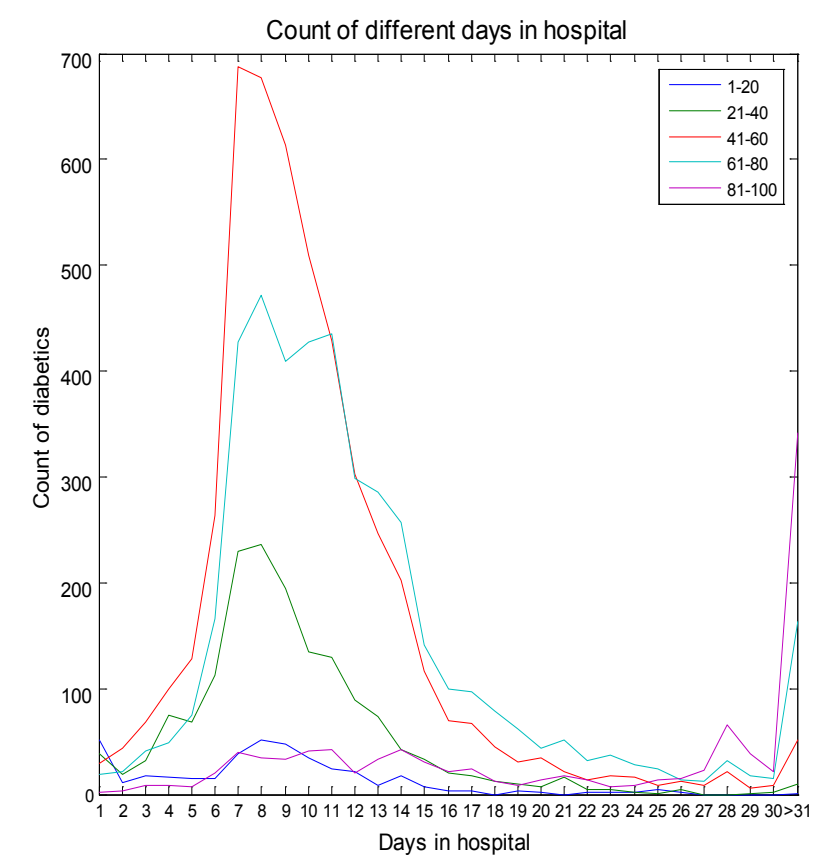

Figure 2a: In hospital, days distribution of diabetics in different age groups. Horizontal axis represents in hospital days, days more than 30 are merged into one group called " $>31$ ". Vertical axis represents the number of diabetics of each in hospital day. Different color lines represent different age groups.

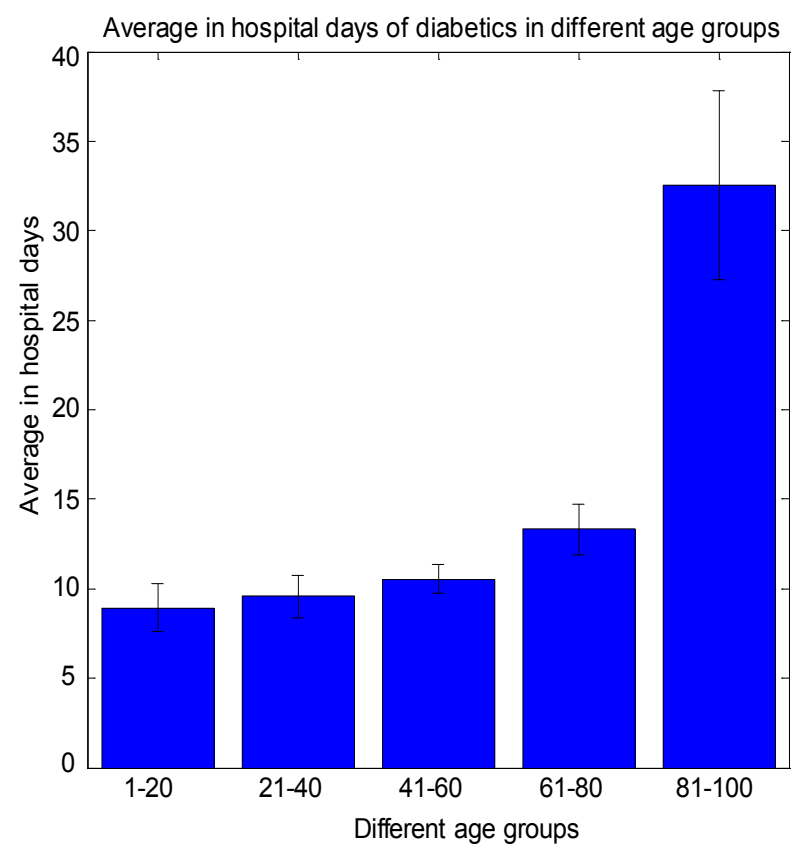

Figure 2b: Average in hospital days of diabetics in different age groups Horizontal axis represents different age-groups. Vertical axis represents mean hospital stay. Error bars are marked with standard deviation. 
Citation: Dai Z, Zhang X, Shan H, Liu Y (2015) Trends in Age Distributions, Complications, Hospital Stay, and Cost Incurred in a Chinese Diabetic Population from 2006 to 2013. J Bioanal Biomed 7: 050-053. doi:10.4172/1948-593X.1000123

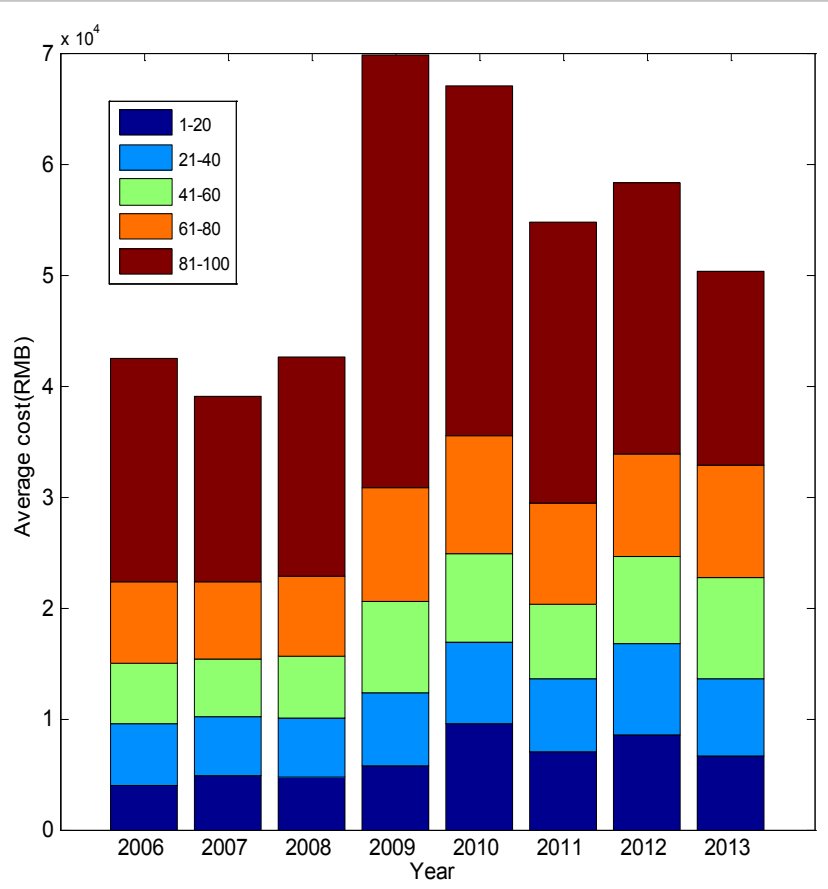

Figure 3a: Hospital cost of diabetics in different age groups. Horizontal axis represents the years from 2006 to 2013. Relatively, the proportion of in hospital days of diabetics is represented by vertical axis in different age groups. Different colors represent different age-groups.

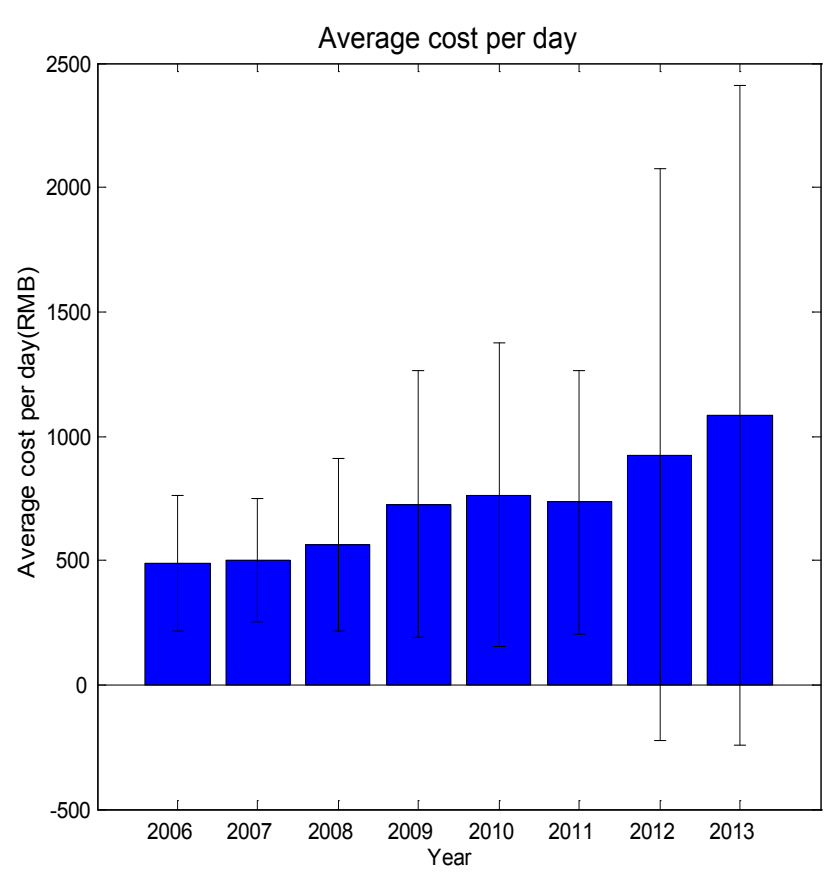

Figure 3b: Average hospital cost of diabetics per day. Horizontal axis represents the years from 2006 to 2013. Vertical axis represents the mean hospital cost per day. Error bars are marked with standard deviation. The cost of each patient is quite different, so the standard deviation is very large.

with four complications spend up to 11,178.0 RMB on average, which increases to 1.9 times compare with the former. It is obvious that along with the increase of complication number, hospital costs are getting higher.

\begin{tabular}{|c|c|}
\hline Complication number & Patient number \\
\hline 0 & 2,128 \\
\hline 1 & 3,039 \\
\hline 2 & 2,580 \\
\hline 3 & 1,846 \\
\hline 4 & 1,145 \\
\hline 5 & 1,418 \\
\hline 6 & 58 \\
\hline
\end{tabular}

Table 1: Complication number of diabetics.

\begin{tabular}{|c|c|c|}
\hline Diagnosis & percent & count \\
\hline diabetic neuropathy & $24.26 \%$ & 769 \\
\hline diabetic nephropathy & $17.54 \%$ & 556 \\
\hline diabetic eye disease & $16.18 \%$ & 513 \\
\hline diabetic ketoacidosis & $11.58 \%$ & 367 \\
\hline diabetic pedipathy & $8.52 \%$ & 270 \\
\hline hyperlipidemia & $6.75 \%$ & 214 \\
\hline fatty liver & $6.62 \%$ & 210 \\
\hline diabetic ketoacidosis & $6.25 \%$ & 198 \\
\hline diabetic peripheral & $2.30 \%$ & 73 \\
\hline vascular lesions & & \\
\hline
\end{tabular}

Table 2: Statistic of diabetic complication.

\begin{tabular}{|c|c|c|}
\hline Complication & percent & count \\
\hline Hypertension & $73.78 \%$ & 3233 \\
\hline infarction & $7.51 \%$ & 329 \\
\hline coronary heart disease & $6.16 \%$ & 270 \\
\hline Thyroid dysfunction & $4.54 \%$ & 199 \\
\hline cataract & $2.26 \%$ & 99 \\
\hline urinary tract infection & $1.96 \%$ & 86 \\
\hline pulmonary infection & $1.32 \%$ & 58 \\
\hline adiposity & $1.30 \%$ & 57 \\
\hline osteoporosis & $1.16 \%$ & 51 \\
\hline
\end{tabular}

Table 3: Statistic of diabetic comorbidity.

We also analyzed comorbidity of diabetics (complication that is not caused by diabetes). Table 3 lists the most 9 comorbidities. We can see that common cardio-cerebrovascular diseases are the main comorbidities.

\section{Analysis by year}

The patient information and hospital cost in different years shows as Table 4. The in hospital days decreased from 14.15 in 2006 to 10.61 in 2013. Hospital cost presented an increasing trend from 2006 to 2009 and decreased from 2009 to 2013 . These are related with better health service, rich medicine and better management on daily hospitalization.

\section{Discussion}

From the analysis above we can see that in China although aged people tend to have higher rates of diabetes [10], the incidence of diabetes in the young people (1-20 years old) is increasing faster $(\mathrm{p}<0.001)$ (Figure 1) than other people (over 20 years old). The rate of people with diabetes under 40 years old is getting higher, which is closely related to the unhealthy lifestyle of modern people. It could be the reason by fast life pace, high life pressure, lack of sleep and sports exercise and unhealthy diets. So, residents should pay attention to their own health and the change of blood glucose, strengthen sports exercises, control their diet, and stay far away from diabetes and impaired fasting glucose. As for people with diabetes, they should pay more attention to 
Citation: Dai Z, Zhang X, Shan H, Liu Y (2015) Trends in Age Distributions, Complications, Hospital Stay, and Cost Incurred in a Chinese Diabetic Population from 2006 to 2013. J Bioanal Biomed 7: 050-053. doi:10.4172/1948-593X.1000123

\begin{tabular}{|c|c|c|c|c|}
\hline Year & Average age & Average days in hospital & Average cost (yuan) & Cost per day (yuan) \\
\hline 2006 & 57.6 & 14.1 & $7,192.4$ & 488.9 \\
\hline 2007 & 57.3 & 13.7 & $6,545.1$ & 499.9 \\
\hline 2008 & 57.0 & 13.0 & $7,028.9$ & 563.0 \\
\hline 2009 & 58.3 & 14.45 & $11,694.4$ & 724.3 \\
\hline 2010 & 57.5 & 14.3 & $10,915.8$ & 762.3 \\
\hline 2011 & 57.0 & 12.6 & $8,975.8$ & 725.13 \\
\hline 2012 & 56.6 & 12.0 & $9,861.5$ & 915.5 \\
\hline 2013 & 56.5 & 10.6 & $9,872.5$ & $1,073.3$ \\
\hline Average & 57.2 & 13.1 & $9,010.8$ & 719.0 \\
\hline
\end{tabular}

Table 4: Analysis in different years.

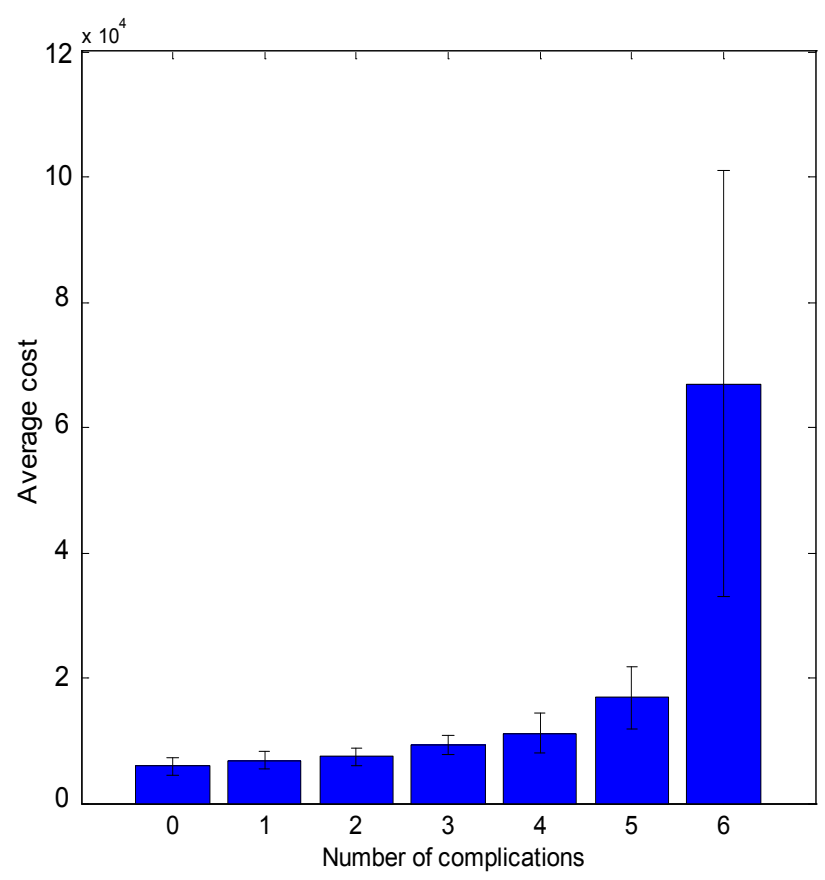

Figure 4: Average cost of diabetics with different number of complications. The numbers of complications is represented by horizontal axis while the daily hospital costs are represented by vertical axis. Error bars are marked with standard deviation.

their dietary structure and do more sports exercise, control blood sugar level and keep in a stable condition.

For diabetes, the days of staying in hospital and hospitalization costs have direct relation to the age. The elder the diabetics are, the more days and costs in hospital. With the basic disease and the decreasing of immune ability in elder patients the number of complications and mean hospitalization cost increased. Average cost in hospital of diabetics with four complications is $11178.0 \mathrm{RMB}$, which is 1.9 times of diabetics without complication. Therefore, elder diabetics should pay attention to physical changes, effectively control blood glucose and stabilize illness condition. In a sense, it can alleviate the economic burden by decreasing the time in hospital.

Meanwhile, hospital stay and cost of diabetics also have close relation to the number of complications. Our results showed that diabetics without complications spend less in hospital than those with complications on things such as medicine, assay, and inspection. The more complications they have and the later complications are discovered, the longer diabetics stay in hospital. In the wake of all other increasing cost such as bed and medicine, hospital stay increases unavoidably. From the above, hospital cost can be effectively controlled by controlling on the number of the complications, so does the economic burden.

In the research data, for the first other diagnosis, common cardiocerebrovascular diseases have 3,928 cases, such as hypertension, hyperlipidemia, fatty liver, coronary heart disease and cerebral thrombosis. It is obvious that common cardio-cerebrovascular diseases are the main comorbidities; in which hypertension have 3,233 cases, accounting for $87.43 \%$. The previous study showed that hypertension has serious impact on the happening and developing of diabetic complications [11]. For the diabetics with hypertension, if hypertension cannot be well controlled, the diabetes can't be either. If hypertension can be well taken care of, hospitalization cost would be greatly lowered.

It can be seen from Table 4 that the average days in hospital decreases while average hospital costs and daily hospital costs increasing. It shows that average time spend on cure is shortened with the improvement in health care, more medicines, and closer hospital management. But in terms of the increasing in costs in hospital, it is clear that government should take various positive measures, including effectively controlling medical prices and alleviating economic burden on diabetics to improve people's health.

\section{Conclusion}

Diabetes has become one of the major diseases that bothered residents' health and caused economic burden [12]. In view of the serious damages brought by diabetes, it should be given high priority by health policy makers, health workers and diabetics in China. Since heredity, diet, obesity, lack of physical exercise and anxiety are factors for diabetes, International Diabetes Federation comes up with best solution from five aspects. The solution involves diet, medicines, tests, physical exercise and physiological treatment and care. Diabetes and high risk groups start from little aspects, by positive prevention and treatment on diabetes and complications. Meanwhile, policy-making sectors should timely make out effective manage policy and control the incidences of diabetes from the development of diabetes, so as to improve the quality of residents' life and lower economic burden on people with diabetes [13].

\section{Acknowledgment}

This work was supported by grants from the National Natural Science Foundation of China (grant numbers 81270952 and 81070684), the Jiangsu Province's Key Provincial Talents Program (BE 2011802), the Projects in the Jiangsu Science and Technology Pillar Program (BE 2011802), the Project Funded by the Priority Academic Program Development of Jiangsu Higher Education Institutions, the Program for Development of Innovative Research Team in the First Affiliated Hospital of NJMU (number 20113012), and the Special Scientific Research Project from the Ministry of Health, China (grant number 201002002). Nanjing Medical University Science and Technology Development Foundation (2012NJMU122). 
Citation: Dai Z, Zhang X, Shan H, Liu Y (2015) Trends in Age Distributions, Complications, Hospital Stay, and Cost Incurred in a Chinese Diabetic Population from 2006 to 2013. J Bioanal Biomed 7: 050-053. doi:10.4172/1948-593X.1000123

\section{References}

1. Shaw JE, Sicree RA, Zimmet PZ (2010) Global estimates of the prevalence of diabetes for 2010 and 2030. Diabetes Res Clin Pract 87: 4-14.

2. Whiting DR, Guariguata L, Weil C, Shaw J (2011) IDF diabetes atlas: global estimates of the prevalence of diabetes for 2011 and 2030. Diabetes Res Clin Pract 94: 311-321.

3. Li R, Lu W, Jiang QW, Li YY, Zhao GM, et al. (2012) Increasing prevalence of type 2 diabetes in Chinese adults in Shanghai. Diabetes Care 35: 1028-1030.

4. Xu Y, Wang L, He J, Bi Y, Li M, et al. (2013) Prevalence and control of diabetes in Chinese adults. JAMA 310: 948-959.

5. Yang W, Lu J, Weng J, Jia W, Ji L, et al. (2010) Prevalence of diabetes among men and women in China. N Engl J Med 362: 1090-1101.

6. Schofield D, Cunich MM, Shrestha RN, Passey ME, Veerman L, et al. (2014) The economic impact of diabetes through lost labour force participation on individuals and government: evidence from a microsimulation model. BMC Public Health 14: 220.

7. Meyers JL, Parasuraman S, Bell KF, Graham JP, Candrilli SD (2014) The high-cost, type 2 diabetes mellitus patient: an analysis of managed care administrative data. Arch Public Health 72: 6
8. Lopez JM, Bailey RA, Rupnow MF, Annunziata K (2014) Characterization of type 2 diabetes mellitus burden by age and ethnic groups based on a nationwide survey. Clinical therapeutics 36: 494-506.

9. Baoer (2010) European Diabetes Prevention Guide. Diabetes World pp. 443448.

10. Nayak BS, Sobrian A, Latiff K, Pope D, Rampersad A, et al. (2014) The association of age, gender, ethnicity, family history, obesity and hypertension with type 2 diabetes mellitus in Trinidad. Diabetes and Metabolic Syndrome: Clinical Research \& Reviews 8: 91-95.

11. Unadike BC, Eregie A, Ohwovoriole AE (2011) Prevalence of hypertension amongst persons with diabetes mellitus in Benin City, Nigeria. Niger J Clin Pract 14: 300-302.

12. Guariguata L, Whiting DR, Hambleton I, Beagley J, Linnenkamp U, et al. (2014) Global estimates of diabetes prevalence for 2013 and projections for 2035 . Diabetes Res Clin Pract 103: 137-149.

13. Wild S, Roglic G, Green A, Sicree R, King H (2004) Global prevalence of diabetes: estimates for the year 2000 and projections for 2030. Diabetes Care 27: 1047-1053. 